\title{
SCIENTIFIC REPORTS

\section{OPEN A Clinically Relevant Functional Model of Type-2 Cardio-Renal Syndrome with Paraventricular Changes consequent to Chronic Ischaemic Heart Failure}

\begin{abstract}
Joanne Clare Harrison ${ }^{*}$, Scott Duncan George Smart, Emma Maria Hinemoa Besley,
\end{abstract} Jessica Renee Kelly, Morgayn Iona Read, Yimin Yao \& Ivan Andrew Sammut

Cardiorenal syndrome, de novo renal pathology arising secondary to cardiac insufficiency, is clinically recognised but poorly characterised. This study establishes and characterises a valid model representative of Type 2 cardiorenal syndrome. Extensive permanent left ventricular infarction, induced by ligation of the left anterior descending coronary artery in Lewis rats, was confirmed by plasma cardiac troponin I, histology and cardiac haemodynamics. Renal function and morphology was assessed 90-days post-ligation when heart failure had developed. The involvement of the paraventricular nucleus was investigated using markers of inflammation, apoptosis, reactive oxygen species and of angiotensin II involvement. An extensive left ventricular infarct was confirmed following coronary artery ligation, resulting in increased left ventricular weight and compromised left ventricular diastolic function and developed pressure. Glomerular filtration was significantly decreased, fractional excretion of sodium and caspase activities were increased and basement membrane thickening, indicating glomerulosclerosis, was evident. Interestingly, angiotensin II receptor I expression and reactive oxygen species levels in the hypothalamic paraventricular nucleus remained significantly increased at 90-days post-coronary artery ligation, suggesting that these hypothalamic changes may represent a novel, valuable pharmacological target. This model provides conclusive morphological, biochemical and functional evidence of renal injury consequent to heart failure, truly representative of Type-2 cardiorenal syndrome.

Cardiorenal syndrome (CRS) is a clinically recognised but poorly characterised condition which is generally divided into multiple subtypes to aid the selection of specialised interventions ${ }^{1}$. Type- 2 CRS describes chronic cardiac dysfunction, such as chronic heart failure (CHF), leading to progressive chronic kidney disease (CKD). This worsening renal function can then accelerate the underlying myocardial dysfunction. Chronic kidney failure has been reported in $68.3 \%$ of heart failure (HF) patients, with reduced creatinine clearance $(\mathrm{CrCl})^{2}$ and decreased estimated glomerular filtration rate (eGFR) ${ }^{3}$ being recognised predictive factors for mortality. Conversely, improved renal function promotes a favourable prognosis in $\mathrm{HF}^{4}$.

Current international clinical guidelines direct that CKD should be viewed as a prevalent condition of varying severity, and press the need for early detection and improved clinical management ${ }^{5}$. Pathological processes implicated in the generation of CKD secondary to HF include: haemodynamic parameters, excessive autonomic nervous system (ANS) efferent output, systemic release of endocrine mediators and proinflammatory cytokines, impairment of endogenous vasodilatory mechanisms, erythropoietin imbalances and anaemia ${ }^{6-12}$. Activation of systemic and renal renin-angiotensin aldosterone system (RAAS) results in increased angiotensin II (AngII) and aldosterone mediated effects on renal electrolyte and water retention, leading to plasma volume expansion and increasing myocardial workload ${ }^{13}$. Renal denervation has been shown to normalize renal angiotensin receptor expression in animal models of CHF and diabetic nephropathy ${ }^{14,15}$. Conversely, the dysfunctional kidney can 
release inflammatory cytokines into the systemic circulation, provoking myocardial damage. Renal injury is also indicated to increase sympathetic activity within the cardiovascular regulatory centres of the midbrain via the renal somatic afferent nerves, altering cardiac structure and function ${ }^{16,17}$. However, the existence of a clinical correlation between co-morbidities does not imply causality. To confirm causality, the direct effect of myocardial injury upon renal function needs to be replicated in a controlled environment, where possible cofounders can be minimized.

Many animal models of Type-2 CRS have utilized unilateral nephrectomy where the renal injury is surgically induced, rather than resulting as a consequence of the $\mathrm{HF}^{18-20}$. Other studies have reported changes in renal function following myocardial infarction (MI), however, there are no working models which produce both de novo renal functional and morphological changes, consistent with CKD directly consequent to chronic HF.

Current recommended treatments for HF, such as angiotensin converting enzyme (ACE) inhibitors and $\mathrm{AT}_{1} \mathrm{R}$ antagonists, mainly act to symptomatically treat the peripheral cardiovascular manifestations of HF. To date, the role of the cardiac control centres of the brain have largely been ignored in the search for new targets to treat HF. However, there is growing evidence that the central nervous system (CNS) plays a major role in the pathophysiology of ischaemic $\mathrm{HF}^{21}$. Severing the pathways connecting the organum vasculosum of the lamina terminalis to the paraventricular nucleus (PVN) of the hypothalamus was found to attenuate characteristic features of $\mathrm{HF}^{22}$ but the mechanisms of activation of the PVN in HF are yet to be clearly elucidated. Current theories include the systemic release of pro-inflammatory cytokines and Ang II from damaged myocardium, and an endothelial release of prostaglandin $\mathrm{E}_{2}$ acting directly on the $\mathrm{PVN}^{23}$. Cardiac neural afferent activation as a consequence of MI has also been directly implicated in the formation of pro-inflammatory cytokines in the PVN of the hypothalamus correlating with an increased activation of microglial cells in this region occurring independently of blood-borne pro-inflammatory cytokine levels ${ }^{24-27}$. Hypothalamic ROS production has also been associated with activation of the sympathetic system and may be implicated in the progression of renal and cardiac dysfunction in HF, as elevated levels of ROS have been observed in the PVN in $\mathrm{HF}^{28-34}$. We sought to examine the involvement of ROS as well as other neuroinflammatory mediators such as TNF- $\alpha$ in $\mathrm{HF}^{33}$.

Previous studies mostly examined PVN changes during the early stages of HF focusing on the four to six week period post coronary artery ligation with only one study examining this region at 56 days post. We therefore investigated if these biomarker changes in the PVN were maintained or even elevated over the course of HF pathogenesis. We also sought to determine if CHF simultaneously provoked changes in the hippocampal arm of the hypothalamic pituitary adrenal axis. Our current study characterizes a reliable animal model, truly representative of Type-2 CRS where chronic cardiac dysfunction at 90-days post-MI results in morphological and functional renal injury and indicates an ongoing involvement for the PVN in this syndrome. This model will enable a clearer definition of the pathology involved and the insights gained could be used to produce more targeted treatments.

\section{Results}

Confirmation of cardiac injury. LAD ligation positioning was optimized in a preliminary study to produce a consistent high rate of LV myocardial scaring of the free wall ( $>20 \%$ wet weight) with acceptable perioperative mortality $(<28 \%)$. There was no difference in whole animal fasted body weight $\left(\mathrm{BW}_{\mathrm{f}}\right)$ between surgical groups. Picrosirius-red staining revealed substantial LV enlargement at 90 -days post-ligation, seen as an increased intraventricular volume compared to sham hearts, with substantial wall thinning and a prominent red stained collagenous scar (Fig. 1a). A significant elevation in plasma cTnI was seen at $4 \mathrm{hr}$ post-ligation compared to sham animals $\left(P<0.05\right.$; Fig. 1b). LAD-ligated animals with $\mathrm{cTnI}$ levels $<2 \mathrm{ng} \cdot \mathrm{ml}^{-1}$ were excluded ( 3 animals) as the myocardial infarct was found to be $\leq 20 \%$ at termination (Supplementary Fig. 1 Correlation between plasma cardiac troponin I (cTnI) at $4 \mathrm{hr}$ post coronary ligation and left ventricular infarct size (\% free wall) measured at 90-days), resulting in a total of $11 \mathrm{LAD}$ animals surviving to 90 -days included in the main study. Total ventricular mass per $100 \mathrm{~g}$ of $\mathrm{BW}_{\mathrm{f}}$ was significantly $(P=0.0027)$ increased in LAD versus sham at the time of sacrifice (Fig. 1c). Average scar size in the LAD group was $24.2 \pm 1.5 \%$ wet weight of the LV mass, including the septum. When individual regions of the myocardium were examined in a subset of rats $(n=4)$, it was found that LV and right free wall mass were significantly $(P=0.0103)$ increased following infarction (Table 1$)$. IL-1 $\beta$ was significantly $(P=0.0082)$ increased in the peri-infarct region of LAD animals (Fig. 1d) at 90-days while no significant differences were seen in IL-4, IL- 6 or IL-10 at this time point. Concentrations of IFN- $\gamma$, and TNF- $\alpha$ for all samples were below the detectable limits of the multiplex assay.

Analysis of isolated LV haemodynamic function at 90 -days showed that LAD ligation significantly $(P=0.0013)$ compromised LVDP (Fig. 1e) and rate of ventricular relaxation $(P=0.0141$; Fig. 1g). Ventricular contractile rates (dP.dt $\mathrm{min}^{-1}$, Fig. 1f) in this surgically ligated group were also decreased $(P=0.0547)$. Spontaneous HR fell $(P=0.023)$ in the ligated animals (Fig. 1h) but no change in coronary flow between sham and LAD groups was observed.

Blood analysis. Arterial blood samples taken at the time of LAD surgery and venous blood samples taken at organ harvest showed no evidence of anaemia, with no significant difference in hemoglobin concentration, or hematocrit, between sham and LAD animals. There was no significant change in $\mathrm{pH}$, circulating electrolyte concentrations $\left(\mathrm{Ca}^{2+}, \mathrm{Na}^{+}\right.$, or $\left.\mathrm{K}^{+}\right)$or oxygen carrying potential of blood samples between groups or time points (see Supplementary Data Table 1).

Impaired renal function and structural injury following CHF. At 90-days post-LAD all animals in the LAD-ligated group showed detrimental changes in renal function and structure. eGFR was decreased with $\mathrm{CrCl}$ reduced to $45 \%$ of the value of the Sham group $(P<0.0001$; Fig. $2 \mathrm{a})$, while $\mathrm{FE}_{\mathrm{Na}}(P<0.001$; Fig. 2b) was significantly increased. Twenty four hour urinary protein levels were almost doubled $(P<0.0001)$ in the LAD-ligated 
a. Level $1 \quad$ Level $2 \quad$ Level 3

Sham
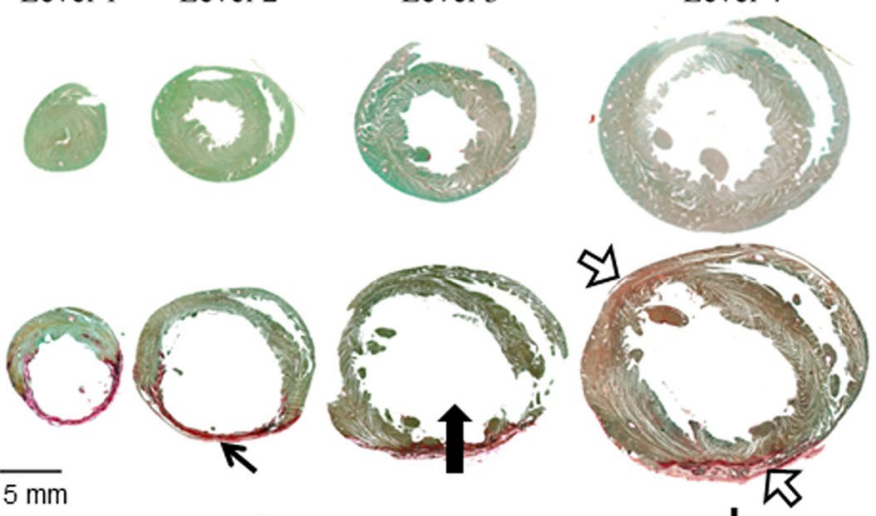

b.

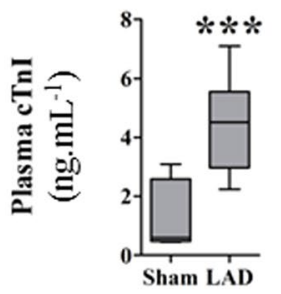

c.

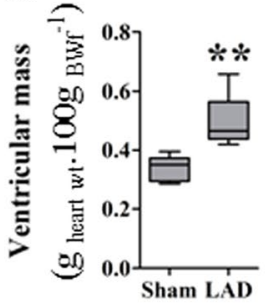

d.

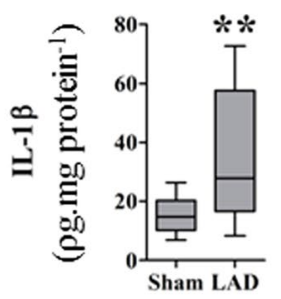

e.

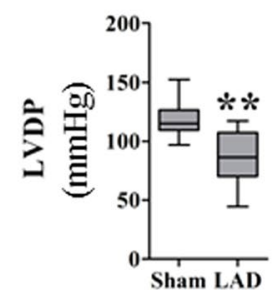

f.

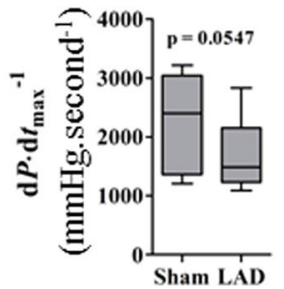

g.

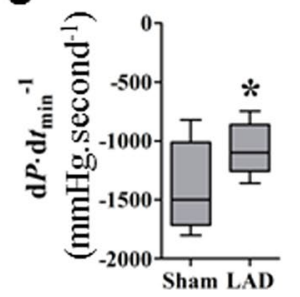

h.

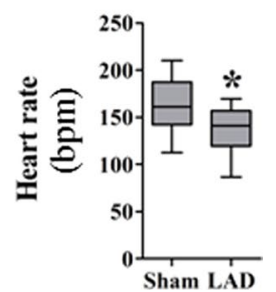

Figure 1. Confirmation of cardiac injury and functional loss 90-days post left anterior descending (LAD) coronary artery ligation: (a) Picrosirius-red stained cardiac sections showing ventricular enlargement $(\rightarrow$ ), wall thinning $(\boldsymbol{K})$ and a prominent red stained collagenous scar $(\Rightarrow)$ in LAD artery ligated hearts compared to sham hearts. Ventricular apex is represented by level one with the base at level four. Box-Whiskers plots showing: (b) plasma troponin (cTnI) concentrations at $4 \mathrm{hr}$ post-ligation, (c) left ventricular mass ( $\mathrm{g}_{\text {heart wt }} \cdot 100 \mathrm{~g}_{\mathrm{BWf}}^{-1}$ ), and (d) IL-1 $\beta$ concentration in the myocardium 90-days post-ligation in sham and LAD animals respectively. Left ventricular function was measured as: (e) left ventricular developed pressure (LVDP), (f) systolic rate of contraction ( $\left.\mathrm{d} P . \mathrm{dt}^{-1} \mathrm{max},\right)$, (g) diastolic rate of relaxation ( $\left.\mathrm{d} P . \mathrm{dt}^{-1} \mathrm{~min}\right)$, and (h) un-paced heart rate (HR), in an isolated ex vivo preparation 90-days post-ligation in sham and LAD animals, respectively $(n=9$ sham $n=9$ LAD). $* P<0.05$, ** $P<0.01, * * * P<0.001$ compared to sham animals.

\begin{tabular}{|l|l|l|l|}
\hline Tissue Region & Sham Mass $(\mathrm{g})$ & LAD Mass $(\mathrm{g})$ & $\boldsymbol{P}$ \\
\hline Left ventricular free wall mass* & $0.53 \pm 0.10$ & $1.03 \pm 0.11$ & 0.0103 \\
\hline Scar & 0.0 & $0.32 \pm 0.02$ & N.A. \\
\hline Septum & $0.42 \pm 0.11$ & $0.32 \pm 0.05$ & 0.2366 \\
\hline Right ventricular free wall & $0.45 \pm 0.04$ & $0.58 \pm 0.04$ & 0.0381 \\
\hline
\end{tabular}

Table 1. Effect of LAD ligation on ventricular scar and wall size. Impact of left anterior descending coronary artery (LAD) ligation on ventricular regional mass at 90 days in a subset of animals ( $n=4 /$ group). *Including infarct scar.

animals (Fig. 2c). Caspase-3/7 activity was significantly increased in both the renal cortex $(P=0.007)$ and to a much greater extent in the medulla $(P=0.0006$; Fig. 2 d,e respectively). LAD surgery significantly $(\mathrm{p}<0.05)$ increased IL- $1 \beta$ concentration in the kidney cortex, from $1.22 \pm 0.14$ to $2.11 \pm 0.14 \mathrm{pg} / \mathrm{mg}$ protein in sham and LAD animals respectively. Elevated levels of profibrotic TGF- $\beta 2$ were identified in the renal medulla (Fig. 2f), although the isoforms, TGF- $\beta 1$ and TGF- $\beta 3$, fell below the detectable limits of the assay at this 90 -day time point. Increased collagen deposition was observed in the glomerular structures and in the tubulointerstitium of the LAD animals (Fig. 3). Glomerulosclerosis, seen as collagen deposition, was observed around the glomerular 
a.

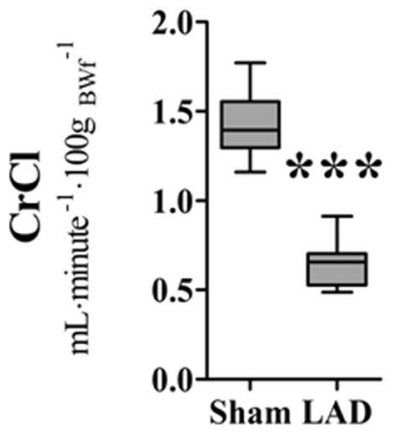

b.

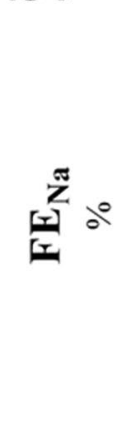

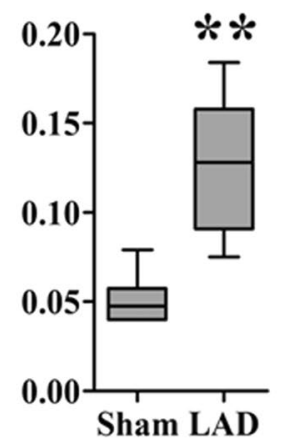

e.
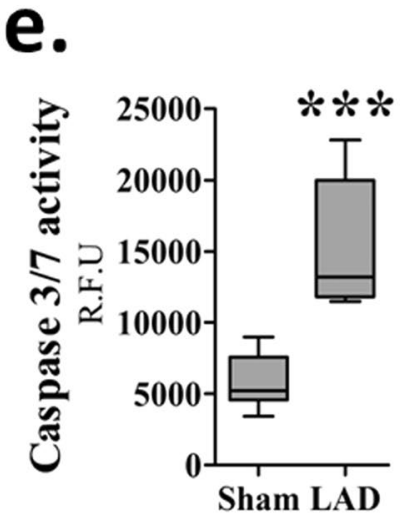

C.

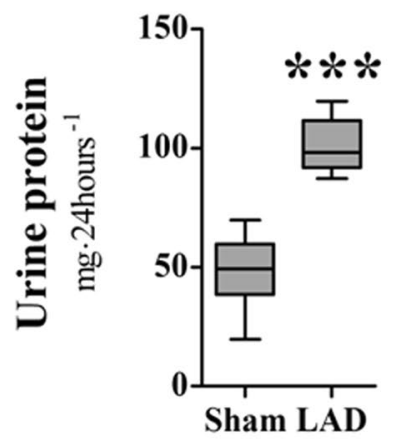

d.

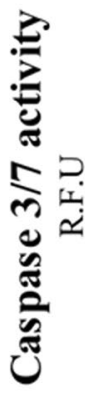

15000

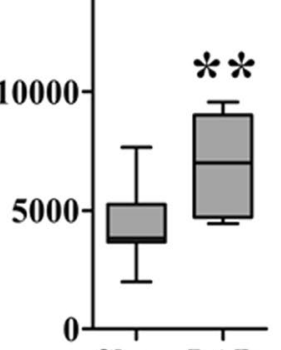

Sham LAD

Figure 2. Left anterior descending (LAD) coronary artery ligation induces renal injury and functional deficits: (a) Calculated creatinine clearance $(\mathrm{CrCl}) . * * * P<0.0001, n=8$ /group. (b) Fractional excretion of sodium $\left(\mathrm{FE}_{\mathrm{Na}}\right)$. $* * P=0.0011, n=6 /$ group. (c) Urinary protein $* * * P<0.0001, n=9 /$ group. (d) Caspase 3/7 activity in the renal cortex $* * P=0.007, n=8$ /group. (e). Caspase $3 / 7$ activity in the medulla $* * * P=0.0006, n=7 /$ group. (f) TGF- $\beta 2$ concentration in the renal medulla $* P=0.0337, n=5 /$ group.

blood vessels and basement membrane. Additionally, interstitial fibrosis was also evident around the proximal, distal and convoluted tubule elements of the renal nephron. Basement membrane thickening and mild mesangial expansion were also seen in the LAD-ligated animals.

Biochemical changes in the hypothalamic PVN and in the hippocampus following CHF. IL- $1 \beta$ and IL-6 levels were higher in the hypothalamic region assayed than in the reference hippocampal tissue (Fig. 4a,b). Neither cytokine was however, detectably altered within either brain region as a consequence of surgery. Levels of TNF- $\alpha$ were not detectable in these brain regions in either sham or following CHF development. Caspase-3/7 activity did not differ significantly across groups or between the two brain regions (data not shown).

$\mathrm{AT}_{1} \mathrm{R}$ expression associated with PVN neurons was increased $(P<0.006)$ in the LAD group (Fig. 5a,c,d). Localisation of the PVN in coronal sections was confirmed using anatomical landmarks (Fig. 5e). ROS production in the PVN was significantly elevated $(P<0.04$; Fig. 5b) as indicated by the increased DHE nuclear staining in the LAD group (Fig. 5f,g). No significant differences were found in the number of Ibal positive cells between LAD and sham groups (data not shown).

\section{Discussion}

This study is the first to conclusively show that induction of a substantial myocardial infarct with impaired cardiac function, directly results in significant renal structural and functional impairment, by 90 -days post-MI. This model, truly representative of Type- 2 cardiorenal syndrome also implicates a role for ongoing elevated ROS in the PVN perpetuating the renal damage observed.

The increased LV mass following LAD artery ligation, with corresponding changes in rates of contraction and relaxation, pressure development and heart rate observed are all consistent with the development of $\mathrm{CHF}^{35,36}$. Cardiac dysfunction was accompanied by evidence of ventricular wall thinning and collagenous scar formation, helping confirm the development of diastolic heart failure. Gross morphological presentations associated with $\mathrm{CHF}$ were observed, including an increased ventricular mass compared to body weight due to enlargement of the LV free wall, combined with increased sphericity of the remodelled hearts ${ }^{35,37,38}$. A similar increase in heart to body weight ratio following MI has been seen in a Type- 2 cardiorenal model ${ }^{39}$, however an earlier study had only reported an increase in LV mass in a small subset of animals ${ }^{40}$, possibly reflecting a difference in the severity of cardiac injury. 


\section{Sham}
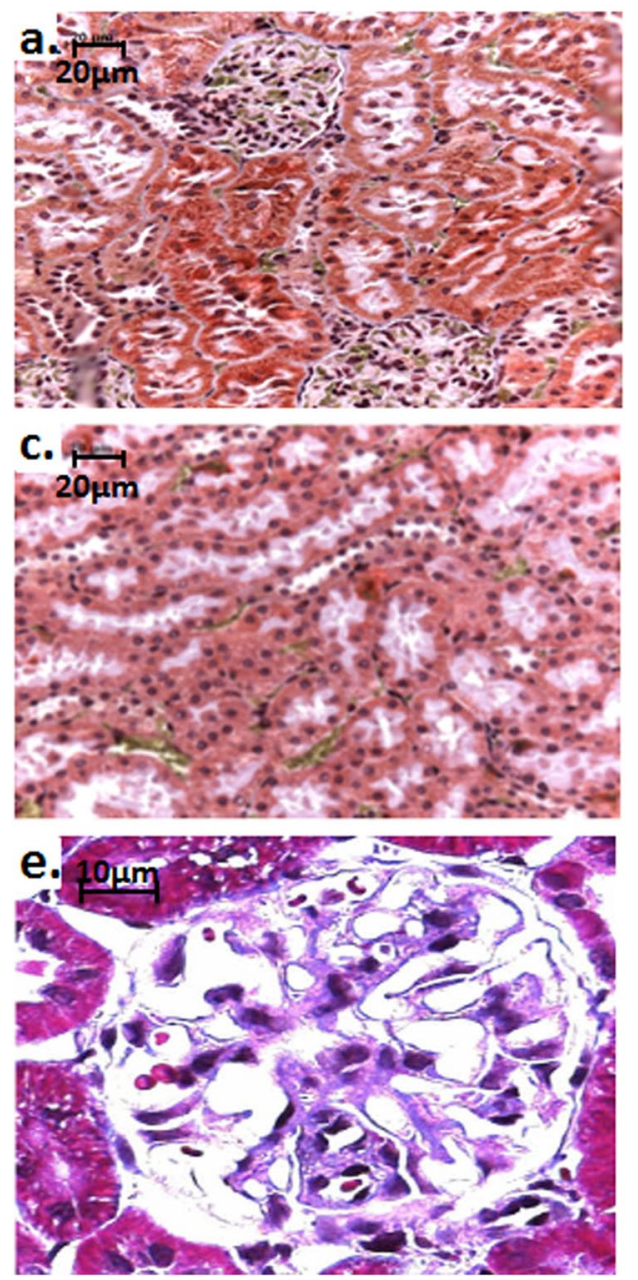

LAD
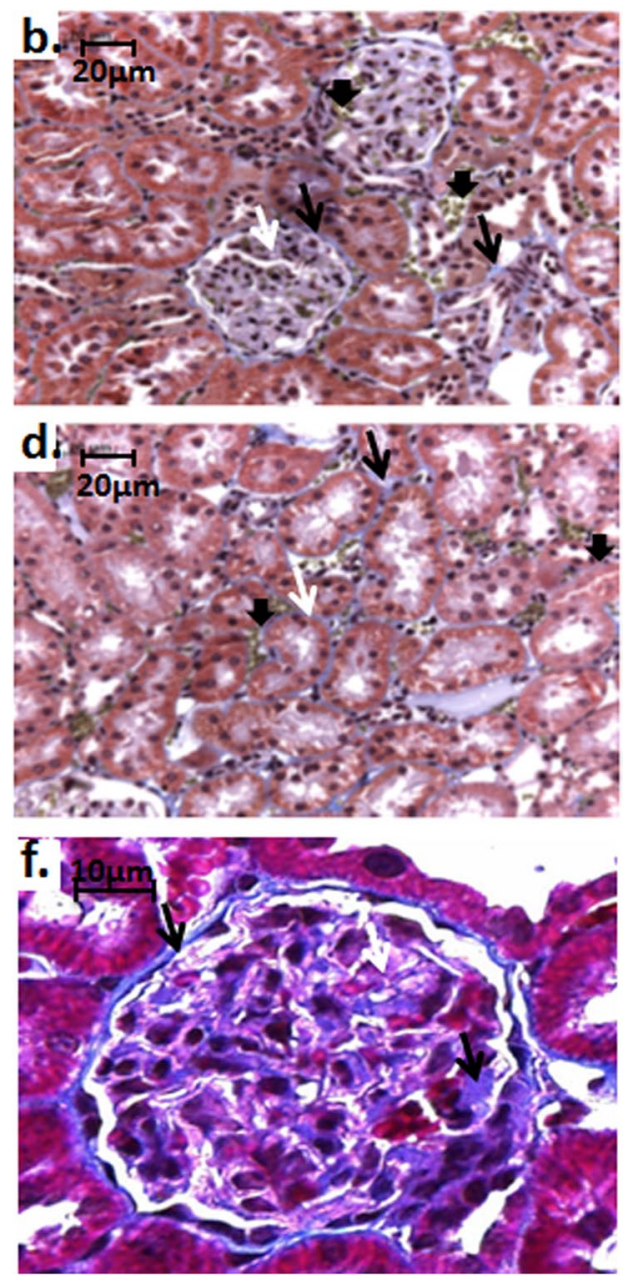

Figure 3. Left anterior descending (LAD) coronary artery ligation increases glomerular and tubulointerstitial collagen deposition and mesangial expansion. Martius Scarlet Blue stained renal cortex and medulla (a-d) Nuclei are stained blue/black, muscle pale red, collagen blue $(\rightarrow)$, fibrin stained red, with early fibrin yellow, $(\downarrow)$ and old fibrin blue $(\Leftrightarrow)$. (a) Renal cortex of a sham operated animal, showing normal morphology. (b) Renal cortex of a LAD-ligated animal, showing increased collagen deposition around glomeruli and in the tubulointerstitium. (c) Renal medulla of a sham operated animal, showing normal morphology. (d) Renal medulla of a LAD-ligated animal, showing increased tubulointerstitial collagen deposition. Masson's trichrome stained renal glomeruli $(\mathbf{e}, \mathbf{f})$. Nuclei are stained blue/black, cytoplasm and muscle are stained red, and collagen is stained blue. (e) Glomeruli of a sham operated animal, showing normal morphology. (f) Glomeruli of LAD ligated animal, showing mesangial expansion $(\hookrightarrow)$ and increased collagen staining $(\rightarrow)$.

The model described here provides conclusive evidence that morphological, biochemical and functional glomerular and tubular injury results directly from a chronic primary cardiac insult truly representative of Type- 2 CRS. Previous similar studies terminated at either 56 days ${ }^{39}$ or 112 days post-MI ${ }^{41,42}$ only produced a modest de novo change in renal morphology ${ }^{39}$ or increased fibrotic and injury markers ${ }^{42}$ secondary to MI but failed to demonstrate any significant changes in renal function. $\mathrm{CrCl}$ was shown to decrease mildly at 56 days post-LAD ligation $^{39}$ and again in another 12 week CHF study ${ }^{43}$, however unlike the present study, no increase in $\mathrm{FE}_{\mathrm{Na}}$, protein urea or tissue pathology were observed. Similarly, the 12 week study failed to demonstrate any changes in tubule function ${ }^{43}$. Our findings show that $\mathrm{CrCl}$ was significantly reduced at 90 -day post-MI, indicative of impaired glomerular perfusion and filtration ${ }^{44}$ and suggests that the degree of glomerular injury recorded is directly related to the duration and extent of CHF development. The small increase in $\mathrm{FE}_{\mathrm{Na}}$ our animals, reinforces the scenario of an early-stage renal decline existing at the termination point. Equally increased urinary protein indicative of tubular dysfunction observed in the LAD occlusion group 90-day post-MI is also consistent with the development of HF and is predictive of the progression of chronic renal disease to end-stage renal disease $\mathrm{e}^{45-48}$. In contrast, the most realistic previously published model of Type-2 CRS lacked this predictive increase in microalbuminuria ${ }^{43}$. The detection of these functional differences in our findings may be attributable to the use of cTnI as a marker of cardiac injury, allowing us to limit our assessments to those animals with severe sizable 
a.
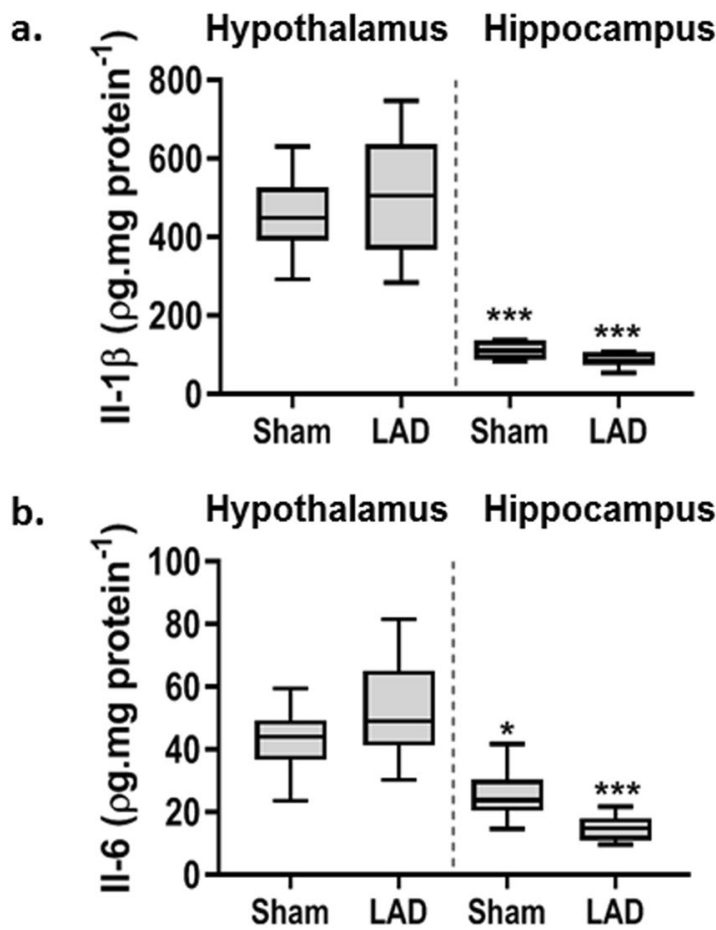

Figure 4. Cytokine levels in the Paraventricular Nucl us consequent to Chronic Ischaemic Heart Failure: (a) IL-1 $\beta$ and (b) IL-6 concentrations were elevated in the hypothalamus compared to control tissue from the hippocampus but no differences were observed between LAD-ligated animals $v s$. sham animals 90 -days post-surgery. Data $=$ mean \pm SEM for each treatment group, $n=6-8$ /group. $* * * P<0.001$ compared to hypothalamus.

infarcts. This refinement is in contrast to the limited histological measurements of infarct size used in the 12 week study. Our observation that the degree of renal injury is proportional to the extent of MI has been seen previously but required a combined insult of diabetes and MI to produce renal functional impairment ${ }^{49}$.

Renal apoptosis has been shown to progressively increase in animal models up to 56 days following MI and has also been clinically attributed to the pathological homeostatic signalling seen in $\mathrm{CHF}^{39,50,51}$. The elevated renal caspase $3 / 7$ activity observed here at 90 -days post-MI indicates that renal apoptosis is still ongoing at this later time point. The finding of proteinuria suggests the development of podocyte injury which is known to trigger fibrosis $^{52}$. A profibrotic model of renal injury developing by 90 -days post-MI is indicated here given the elevated levels of renal TGF- $\beta 2$ detected ${ }^{39,41}$. Morphological changes, consistent with fibrotic remodelling subsequent to apoptosis, were confirmed through histological analysis showing increased collagen deposition in the tubulointerstitium and in the glomerular space, consistent with glomerulosclerosis.

Haemoglobin levels were not perturbed; therefore it is unlikely that the renal dysfunction observed was severe enough to disrupt normal erythropoietin signalling and red blood cell turnover. There was no systemic disturbance of blood electrolytes, which is consistent with the renal dysfunction occurring as a secondary event to $\mathrm{CHF}$, rather than as a consequence of electrolyte imbalance. The lack of changes in methaemoglobin (metHb) or fraction of carboxyhaemoglobin ( $\mathrm{fCOHb}$ ) levels indicates that the oxygenation potential of the whole blood had not been compromised. While anaemia is an important prognostic factor in cardiorenal-anaemia syndrome, it was not present in this Type- 2 cardiorenal model ${ }^{53,54}$.

An ongoing inflammatory response in close proximity to areas of renal fibrosis has been observed up to 56 days post-LAD occlusion ${ }^{39}$. However, in the present study IL- $1 \beta$ in the renal cortex was the only cytokine other than TGF- 32 , of the six measured at 90 -days post-infarction, which was significantly altered. The general lack of a cytokine response may be indicative of resolution of any inflammatory response occurring prior to tissue harvest or it may be due to a tissue dilution effect as the entire medulla or cortex were assayed. IL-1 $\beta$ is known to play a role in infarct healing and is a modulator of pro-fibrotic $\mathrm{AT}_{1} \mathrm{R}$ pathways, supporting a hypothesis of resolving inflammation ${ }^{55,56}$.

Many clinical pharmacological interventions employed in CHF, such as beta blockers, may coincidentally reduce the development of renal injury ${ }^{57}$, when the myocardial damage and subsequent renal dysfunction are less severe $^{58,59}$. Renal hypoxia, however, also triggers a cascade of intrarenal signalling which can lead to an overall change in the renal landscape. Mediators, such as catecholamines, Ang II, ROS and nitric oxide ${ }^{42,60-62}$ represent therapeutic targets which have shown some success in minimising disease progression when pharmacologically blocked $^{58,61,63-65}$. CKD, however remains a major risk factor in CHF patients with reduced cardiac output and novel therapeutic targets are still needed to address the pathological activation of systemic and renal RAAS which promote electrolyte retention and plasma volume expansion ${ }^{13}$. Kidney injury in Type-2 CRS therefore most likely 
a.

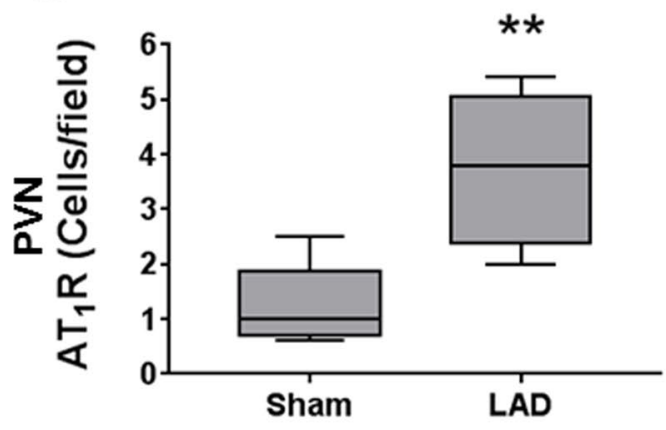

b.

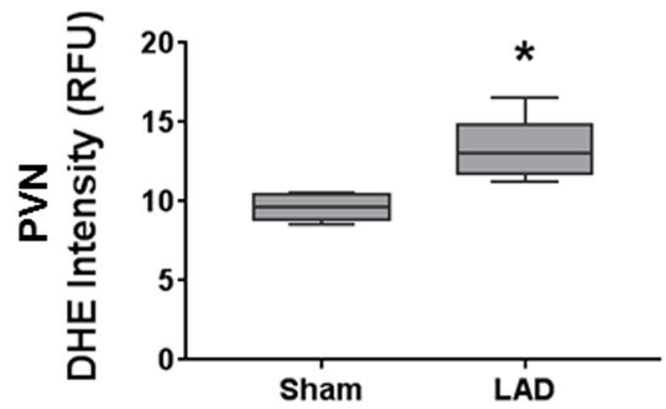

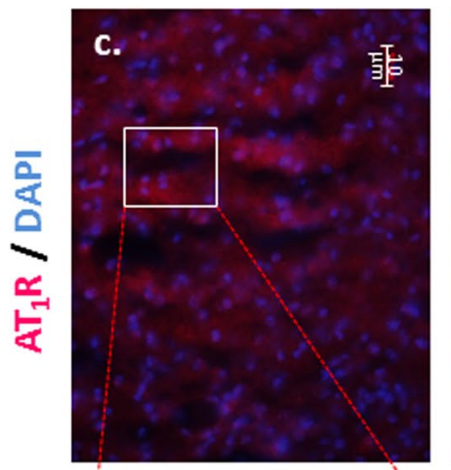
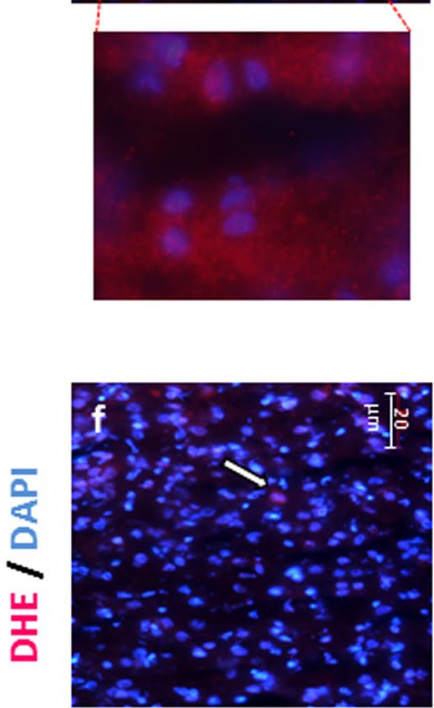
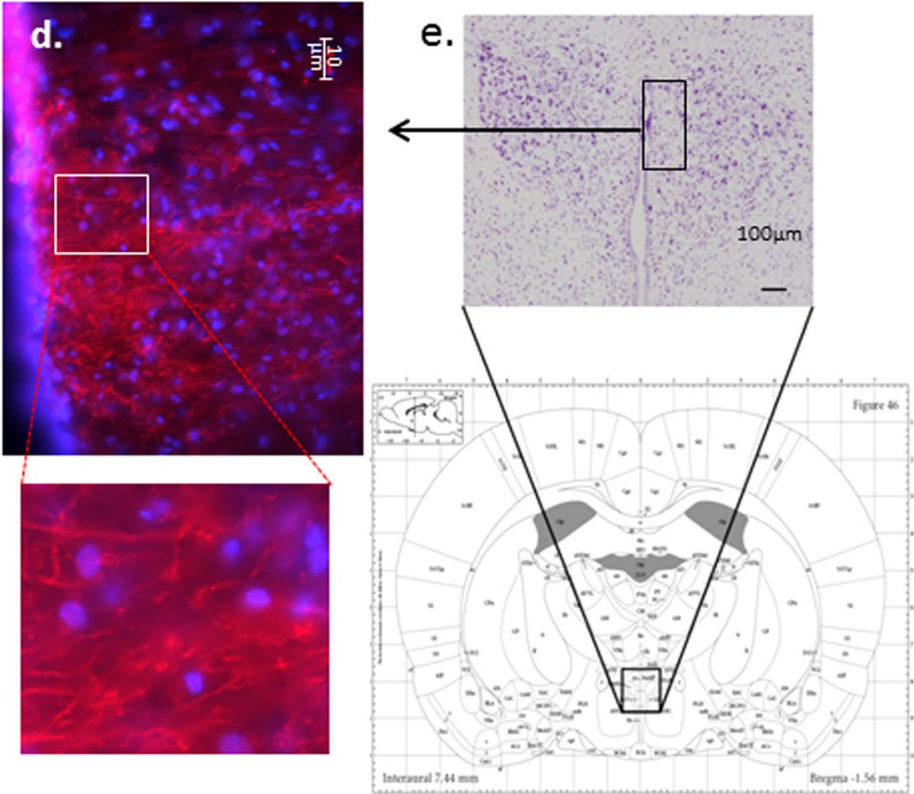

Figure 5. Paraventricular Nuclear changes consequent to Chronic Ischaemic Heart Failure: (a) $A_{1} R_{1}$ expression in the paraventricular nucleus (PVN). The number of $\mathrm{AT}_{1} \mathrm{R}$-positive cells per field counted in 6 sections per animal, $n=5$ animals per treatment group. $* * P<0.005$ compared to sham group. (b) DHE fluorescence indicative of ROS production was measured in 4-6 sections per animal, $n=5 /$ animals per treatment group. $* P<0.05$ compared to sham group. Data is presented as mean \pm SEM for each treatment group. $\mathrm{AT}_{1} \mathrm{R}$ localisation in the anterior PVN co-stained with the nuclear DAPI marker from (c) sham and (d) LAD ligated animals at 3 months post-surgery (scale bar $=10 \mu \mathrm{m}$ ) with inset figures representing a magnified $(\times 3)$ subsection. Localisation of PVN in coronal sections (e) using anatomical landmarks, section stained with cresyl violet for illustrative purposes. Merged DHE fluorescent images showing ethidium co-localisation to DAPI-stained nuclei (indicated by $\Rightarrow$ ) in the PVN of (f) sham showing DAPI nuclear staining and (g) LAD ligated animals with predominately DHE stained nuclear fluorescence at 3 months as a measure of ROS induced cleavage of DHE (scale bar $=20 \mu \mathrm{m})$.

ensues from the combined effects of renal hypoperfusion, with an excessive ANS afferent output, and the systemic release of endocrine mediators and proinflammatory cytokines. This renal injury can subsequently increase posterior hypothalamic noradrenaline turnover and renal SNA elevation ${ }^{66}$. 
This model of Type-2 CRS has enabled preliminary investigations of the interplay of these hypothalamic mediators in this chronic condition. The functional importance of an acute increase in pro-inflammatory cytokines in the PVN 14 days post-MI has been established in an animal model of $\mathrm{HF}^{34}$. However, pro-inflammatory cytokines were not significantly elevated in the PVN in the current study indicating this cytokine response may no longer be important by 90 -days post-MI. ROS levels, however, were still elevated in the PVN and may be an important mediator of the ongoing renal injury. In a model of high salt-induced hypertension, ROS in the PVN affected renal sympathetic nerve activity, resulting in elevated plasma norepinephrine and mean arterial pressure $^{67}$. Increased sympathetic nerve outflow in response to activation of pro-renin receptors in the PVN also appears to involve a ROS mediated signalling pathway ${ }^{68}$. Elevated oxidative stress in the PVN has been previously reported at 28 days post MI, probably due to $\mathrm{AT}_{1}$ receptor mediated NF- $\kappa \mathrm{B}$ signalling resulting in an imbalance of neurotransmitters contributing to sympathoexcitation. Both the oxidative stress and extent of myocardial injury were reduced by bilateral PVN infusion of losartan ${ }^{69}$. Components of brain RAAS, including $\mathrm{ACE}^{70}$ and $\mathrm{AT}_{1} \mathrm{R}^{71}$, are also upregulated early following myocardial injury in several brain regions, including the PVN. Increased expression of the AngII producing enzymes renin and ACE in the PVN seen in HF studies indicates that the increased levels of AngII are produced locally and are not of systemic origin. Upregulation of $\mathrm{AT}_{1} \mathrm{R}$ expression in the PVN of LAD artery ligated animals may be a mechanism by which autonomic and neuroendocrine neurons are recruited in response to the stress of HF. The functional significance of upregulated brain RAAS in models of HF have been confirmed using angiotensinogen antisense RNA which attenuated cardiac remodelling, reduced sympathetic nerve activity and normalised the cardiac reflex responses ${ }^{70}$. The role of AngII was further confirmed by the central administration of the ACE inhibitor, enalaprilat, for 28 days following coronary artery ligation ${ }^{71}$. Enalaprilat attenuated renal responses to acute HF, including increased sodium retention, reduced urine sodium and volume and increased sympathetic activity with impaired baroreflex regulation. Similar results were observed for $\mathrm{AT}_{1} \mathrm{R}$ antagonists. These observations open up the possibility that chronic blockade of either ROS, RAAS or the resulting inducible nitric oxide synthase (iNOS) in the PVN may prevent end organ damage in hypertension or Type-2 CRS.

This model provides a robust methodology to produce Type-2 CRS in a rat, enabling the ability to dissect, in depth, the transition from CHF, to renal injury with the potential to uncover other factor in this complex series of pathologies. However, the current study could not establish a cause-effect relationship between the PVN changes and renal dysfunction. Further studies planned to include pharmacological interventions are required. Initial studies by our group to explore the involvement of RAAS using losartan suggest a reno-protective action for this $\mathrm{AT}_{1} \mathrm{R}$ antagonist in this model. While analysis of cardiac contractility indices were specifically conducted in isolated isovolumic hearts at termination to remove the additional complications of cardiac innervation, future in vivo echocardiographic assessments conducted throughout the experimental time course would provide a valuable assessment of CHF development.

Summary. The use of plasma cTnI to guide the positioning and extent of cardiac injury induction has enabled the establishment and refinement of a model demonstrating all the functional and morphological changes characteristic of Type- 2 cardiorenal syndrome. This clinically-relevant model has also indicated a PVN involvement in the cardiorenal syndrome occurring up to 90-days post-MI, which may represent a distinct pharmacological target for this prevalent condition.

\section{Methods}

Animal care. Inbred male Lewis rats obtained from the University of Otago's Animal Facility were utilised to reduce individual variation in responses. All procedures described were carried out under institutional approval in accordance with the 'Guidelines on the Care and Use of Laboratory Animals' set out by the University of Otago Animal Ethics Committee.

Generation of post-MI chronic HF. Male Lewis rats (275-285 g) were randomly assigned to two main surgical intervention groups, non-ischaemic surgical controls (sham-ligated animals) or cardiac ischaemia by LAD coronary- ligation (LAD-ligated animals) induced, as previously described ${ }^{72-74}$. Animals were intubated under halothane anaesthesia and maintained on a small animal ventilator (SAR-830, Stoelting Co, Chicago USA) with a mix of $70 \% \mathrm{O}_{2} 30 \% \mathrm{NO}_{2}$ and $1-4 \%$ halothane, with flow maintained at $2.3 \mathrm{~mL}$ tidal volume. Core body temperatures were maintained at $37.0 \pm 1{ }^{\circ} \mathrm{C}$ using a warming pad (Kent Scientific, USA). Cannulation of the femoral artery enabled blood sampling for gas analysis at the start of surgery (Radiometer ABL800 Flex blood gas analyser). Permanent coronary artery ligation was achieved using a 7-0 prolene suture around the proximal $\mathrm{LAD}$ artery and the occlusion verified on observation of anterior LV blanching. Bupivicaine $(0.1 \mathrm{~mL})$ was injected around the thoracic incision for post-operative local anaesthesia. Myocardial damage following coronary ligation was confirmed four hours post-surgery by the presence of elevated plasma levels of cardiac troponin-I (cTnI), using a commercially available high sensitivity rat cTnI ELISA (Life Diagnostics, Pennsylvania USA). Surviving LAD-ligated and sham-ligated control animals ( $n=9$ per group) were maintained for 90 -days.

Tissue and sample collection. A heparinised tail vein (IV $-0.5-1.0 \mathrm{~mL})$ blood sample was taken 4 hours after the ligation, or sham ligation and the plasma separated by centrifugation $\left(2,200 \mathrm{~g}\right.$, for 5 minutes, at $\left.4{ }^{\circ} \mathrm{C}\right)$. A 24 hour urine sample was collected using a metabolic cage for 24 hours prior, and for blood gas analysis a blood sample was taken from the inferior vena cava immediately prior to sacrifice. On day 90, animals were weighed, heparinised (Multiparin ${ }^{\circledR}, 500$ USP units $/ \mathrm{kg}$ ) and the hearts excised under halothane anaesthesia and rapidly perfused for isolated haemodynamic assessments ${ }^{74-76}$.

Both kidneys were immediately perfused in situ with $60 \mathrm{~mL}$ of tris-mannitol-sucrose (TMS) buffer containing in mM: 225 mannitol, 75 sucrose, 10 tris $\mathrm{HCl}$ and 0.1 EDTA (dipotassium salt) and $0.1 \mathrm{mM}$ phenyl 
methylsulfonylfluoride protease inhibitor $\left(\mathrm{pH} \mathrm{7.2,} 4^{\circ} \mathrm{C}\right)$. The left kidney was isolated, weighed and divided into cortex and medulla. The hypothalamus and hippocampus (control tissue) were dissected from the brain and cooled in ice-cold TMS buffer. Brain and right kidney tissues were immediately snap-frozen, ground under liquid $\mathrm{N}_{2}$ and stored at $-80^{\circ} \mathrm{C}$ for biochemical analysis. The right kidney was concurrently perfusion-fixed ( $4 \%$ paraformaldehyde) and prepared for histological examination. For immunohistochemistry, forebrains from an additional group ( $n=5$ /group) of animals were prepared for cryosectioning according to standard protocols. Hearts from a subgroup of rats ( $n=5$ /group) were arrested in diastole and ex vivo perfusion fixed in $0.4 \%$ paraformaldehyde. Researchers were blinded to the identity of all post-mortem samples until analysis was completed.

Langendorff haemodynamic assessment. Hearts were cannulated through the aorta and perfused with oxygenated $\left(95 \% \mathrm{O}_{2} / 5 \% \mathrm{CO}_{2}\right)$ Krebs-Henseleit buffer at a constant pressure of $100 \mathrm{~cm} \mathrm{H}_{2} \mathrm{O}$ at $37^{\circ} \mathrm{C}$, in the Langendorff mode. Using a balloon inserted into the LV, developed pressure (LVDP) was calculated as the difference between peak systolic pressure and end diastolic pressure and used to derive $\mathrm{d} P / \mathrm{dtmax}, \mathrm{d} P / \mathrm{dtmin}$ and heart rate (HR) (Chart v5.4.2 software, AD Instruments, Castle Hill, Australia), as previously described ${ }^{74-76}$. LV tissue was subsequently processed for protein and enzyme analysis and stored at $-80^{\circ} \mathrm{C}$. Heart weight was recorded for all animals and in a subset of rats $(n=4)$ the whole heart was dissected into individual left and right ventricles, free walls, septum and scar tissue (if present) then weighed.

Cardiac morphology at $\mathbf{9 0}$-days. The ventricles were sliced coronally into four sequential blocks at $1.5 \mathrm{~mm}$ intervals. Blocks were fixed in Bouin's solution $\left(1 \mathrm{hr} / 55^{\circ} \mathrm{C}\right)$, embedded and $10 \mu \mathrm{m}$ sections cut and stained with picrosirius-red with Fast Green contrast, to produce a strong distinction between viable myocardium (blue green) and collagenous scar tissue (red).

Assessment of renal function. $\mathrm{Na}^{+}$concentrations were measured by flame photometry (FP20 SEAC, Italy) in duplicate urine samples prepared in a $7.5 \mathrm{mM} \mathrm{Li}_{2} \mathrm{CO}_{3}\left(\mathrm{Li}^{+}\right.$concentration of $\left.15 \mathrm{mEq} / \mathrm{L}\right)$. Endogenous creatinine in plasma and urine was analysed using a modified colorimetric Jaffe method and assessed using a Synchron Cx7 Beckman Analyser ${ }^{44}$. GFR was estimated (eGFR) using the calculated CrCl. Fractional excretion of sodium $\left(\mathrm{FE}_{\mathrm{Na}}{ }^{+}\right.$was calculated as the $\left(\right.$Urine $\left[\mathrm{Na}^{+}\right] \times$Plasma $\left.[\mathrm{Cr}]\right) /\left(\right.$ Plasma $\left[\mathrm{Na}^{+}\right] \times$Urine $\left.[\mathrm{Cr}]\right) \times 100$.

Biochemical analyses. Renal cortex and medulla tissue samples were homogenised, using a glass-glass homogeniser, and hypothalamus and hippocampus using a sonicator (Sonics Vibra Cell, John Morris Scientific Ltd, Christchurch, NZ) in ice-cold TMS. Samples were centrifuged $\left(4,680 \mathrm{~g}, 5 \mathrm{mins}\right.$ at $\left.4^{\circ} \mathrm{C}\right)$ and the supernatant processed for ELISA, Milliplex ${ }^{\mathrm{TM}}$ and enzyme activity assays. Results were standardised to sample protein concentrations quantified using a BioRad DC Protein Assay kit (Bio-Rad Laboratories Inc., NZ) ${ }^{77}$. The same protein assay was used to determine urinary protein levels.

Multiplex assays (Milliplex ${ }^{\mathrm{TM}}$ Rat Cytokine Assay kit, Millipore ${ }^{\mathrm{TM}}$, MA, USA) for cardiac (IL-1 $\beta$, IL-4, IL-6, IL-10 and TNF- $\alpha$ ); renal (TGF- $\beta$ and TNF- $\alpha$ ); and brain (IL-1 $\beta$, TNF- $\alpha$ and IL-6) cytokines were performed on homogenate supernatants isolated from peri-infarct cardiac tissue, renal cortex and medulla, and the hypothalamic and hippocampal brain regions, respectively. Samples were subjected to ultra-filtration $(13,000 \mathrm{~g}$ for $1 \mathrm{hr})$ using $0.22 \mu \mathrm{m}$ cellulose acetate filters (Spin-X tubes; Bonnet Equipment, Auckland, NZ) prior to assaying. The assay was conducted according to manufacturer's instructions, with fluorescence emissions from duplicate samples and standards read on a Luminex $100^{\mathrm{TM}}$ analyser (Luminex Corp., TX, USA).

Caspase- 3 and -7 activities were assessed using a homogenous 7 -amino-4-trifluoromethylcoumarin (AFC) caspase- 3 assay kit (Anaspec EnsoLite ${ }^{\mathrm{TM}}$, San Jose, CA, USA) according to the manufacturer's instructions, as previously described ${ }^{75}$. The resultant generation of the AFC fluorophore after an $18 \mathrm{hr}$ incubation was detected using a Gemini-EM fluorometric plate reader (Molecular Devices Corporation, CA, USA) at excitation/emission wavelengths of $380 \mathrm{~nm} / 500 \mathrm{~nm}$, with results expressed as relative fluorescent units per $\mathrm{mg}$ of protein $/ 18 \mathrm{hr}$.

Histology and immunohistochemistry. Collagen fibre accumulation was visualized in renal tissue using Martius Scarlet Blue stain. All qualitative histology evaluations were performed by separate researchers in a blinded manner on 5 individual sections from each block and photographed.

Coronal cryosections $(16 \mu \mathrm{m})$ were cut (Leica, Houston, TX, USA) through the PVN region. Activated microglia were detected using anti-ionized calcium binding adaptor molecule 1 (Iba1) antibody (1:100; Abcam, Sapphire Biosciences, Hamilton, NZ) and AlexaFluor 488 donkey anti-goat IgG secondary antibody (Molecular

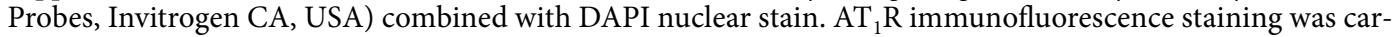
ried out as above using rabbit anti-AT $\mathrm{R}_{1}$ (1:200; Molecular Probes, Invitrogen, CA, USA) and AlexaFluor594 donkey anti-rabbit IgG (1:500; Molecular Probes, Invitrogen, CA, USA). The specificity of the primary antibodies was confirmed by the use of negative control sections in which the primary Ibal and $\mathrm{AT}_{1} \mathrm{R}$ antibodies were not applied. Superoxide production, an indicator of ROS, within the PVN was determined using dihydroethidium (DHE) microfluoroscopy, as described ${ }^{32}$. For each treatment group, a negative control slide was prepared in parallel with no DHE applied. Following 30 mins incubation with DHE $(2 \mu \mathrm{M}$; Molecular Probes, CA, USA $)$ and washing, ProLong Gold Antifade with DAPI nuclear stain (Molecular Probes, Invitrogen, CA, USA) was applied to each section.

For each tissue slice, eight fields from the area of interest were randomly selected around the ventricle by a blinded observer. Iba1, $\mathrm{AT}_{1} \mathrm{R}$ and their respective DAPI images were photographed using a Zeiss LSM 710 confocal microscope (Carl Zeiss Vision, Germany) and overlaid using Adobe Photoshop CS3 software (Adobe). Ibal positive cells were identified and counted using Image J (Version 1.42q, NIH), with both fluorescence and activated microglia morphology taken in to consideration with merged AT1R and Iba1 staining. Ethidium fluorescence, indicative of superoxide formation, was imaged using laser excitation at $568 \mathrm{~nm}$ and fluorescence product 
detected using a $590 \mathrm{~nm}$ long-pass emission filter ${ }^{32}$. Image analysis was performed using Image J and expressed against background controls (no DHE) for each sample.

Statistical analysis. Power analysis utilising error values from previous LAD occlusion studies by the group indicated a requirement of a minimum of $n=8$ /group for functional assays and $n=5 /$ group for biochemical assays. Statistical analysis was performed using Prism ${ }^{\mathrm{TM}} 5$ using a one-tailed unpaired t-test with Welch's correction, with statistical significance taken at $P<0.05$. Results were presented as mean \pm the standard error of the mean (mean $\pm S E M)$. Statistical outliers in biochemical data were removed following a Grubbs' test.

\section{Data availability}

Data and associated protocols are available on request.

Received: 16 October 2019; Accepted: 24 December 2019;

Published online: 27 January 2020

\section{References}

1. Ronco, C., Haapio, M., House, A. A., Anavekar, N. \& Bellomo, R. Cardiorenal syndrome. J. Am. Coll. Cardiol. 52, 1527-1539 (2008).

2. Pons, F. et al. Mortality and cause of death in patients with heart failure: findings at a specialist multidisciplinary heart failure unit. Rev. Esp. Cardiol. 63, 303-314 (2010).

3. Smith, G. L. et al. Renal impairment and outcomes in heart failure: systematic review and meta-analysis. J. Am. Coll. Cardiol. 47, 1987-1996 (2006).

4. de Silva, R. et al. Incidence of renal dysfunction over 6 months in patients with chronic heart failure due to left ventricular systolic dysfunction: contributing factors and relationship to prognosis. Eur. Heart J. 27, 569-581 (2006).

5. Eckardt, K. U. et al. Evolving importance of kidney disease: from subspecialty to global health burden. Lancet. 382, 158-169 (2013).

6. Mullens, W. et al. Importance of venous congestion for worsening of renal function in advanced decompensated heart failure. J. Am. Coll. Cardiol. 53, 589-596 (2009).

7. Oda, Y. et al. Renal insufficiency coexisting with heart failure is related to elevated sympathetic nerve activity. Auton. Neurosci. 155, 104-108 (2010).

8. Park, Y. et al. Increased severity of renal ischemia-reperfusion injury with venous clamping compared to arterial clamping in a rat model. Surgery. 143, 243-251 (2008).

9. Ronco, C. \& Maisel, A. Volume overload and cardiorenal syndromes. Congest. Heart Fail. 16, Si-iv (2010).

10. Testani, J. M. et al. Effect of right ventricular function and venous congestion on cardiorenal interactions during the treatment of decompensated heart failure. Am. J. Cardiol. 105, 511-516 (2010).

11. Unger, T. \& Li, J. The role of the renin-angiotensin-aldosterone system in heart failure. J. Renin Angiotensin Aldosterone Syst. 5, S7-10 (2004).

12. Xu, K. et al. Erythropoietin derivate improves left ventricular systolic performance and attenuates left ventricular remodeling in rats with myocardial infarct-induced heart failure. J. Cardiovasc. Pharmacol. 56, 506-512 (2010).

13. Shotan, A. et al. Fluid overload contributing to heart failure. Nephrol. Dial. Transplant. 20, vii24-vii27 (2005)

14. Clayton, S. C., Haack, K. K. \& Zucker, I. H. Renal denervation modulates angiotensin receptor expression in the renal cortex of rabbits with chronic heart failure. Am. J. Physiol. Ren. Physiol. 300, F31-F39 (2011).

15. Yao, Y. et al. Chronic bilateral renal denervation attenuates renal injury in a transgenic rat model of diabetic nephropathy. Am. J. Physiol. Ren. Physiol. 307, F251-F262 (2014).

16. Goldsmith, S. R., Sobotka, P. A. \& Bart, B. A. The Sympathorenal Axis in Hypertension and Heart Failure. J. Card. Failure. 16, 369-373 (2010).

17. Thaung, H. P. et al. Chronic bilateral renal denervation reduces cardiac hypertrophic remodelling but not beta-adrenergic responsiveness in hypertensive type 1 diabetic rats. Exp. Physiol. 100, 628-639 (2015).

18. Homma, T. et al. Activation of renal angiotensin type 1 receptor contributes to the pathogenesis of progressive renal injury in a rat model of chronic cardiorenal syndrome. Am. J. Physiol. Ren. Physiol. 302, F750-F761 (2012).

19. van Dokkum, R. P. et al. Myocardial infarction enhances progressive renal damage in an experimental model for cardio-renal interaction. J. Am. Soc. Nephrol. 15, 3103-3110 (2004).

20. Tang, S. Y., Peng, D. F., Hu, Y. J. \& Chen, J. Protective effects of valsartan and benazepril combined with atorvastatin on cardiorenal syndrome in rats. Eur. Rev. Med. Pharmacol. Sci. 19, 759-766 (2015).

21. Leenen, F. H. H. Brain Mechanisms Contributing to Sympathetic Hyperactivity and Heart Failure. Circ. Res. 101, 221-223 (2007)

22. Francis, J. et al. Forebrain-mediated adaptations to myocardial infarction in the rat. Am. J. Physiol. Heart Circ. Physiol. 282, H1898-H1906 (2002).

23. Yin, J. et al. Mitochondria-Produced Superoxide Mediates Angiotensin II-Induced Inhibition of Neuronal Potassium Current. Am J Physiol Cell Physiol.ajpcell (2010).

24. Francis, J., Chu, Y., Johnson, A. K., Weiss, R. M. \& Felder, R. B. Acute myocardial infarction induces hypothalamic cytokine synthesis. Am. J. Physiol. Heart Circ. Physiol. 286, H2264-H2271 (2004).

25. Badoer, E. Microglia: activation in acute and chronic inflammatory states and in response to cardiovascular dysfunction. Int. J. Biochem. Cell Biol. 42, 1580-1585 (2010).

26. Rana, I. et al. Microglia activation in the hypothalamic PVN following myocardial infarction. Brain Research. 1326, 96-104 (2010).

27. Shi, P., Raizada, M. K. \& Sumners, C. Brain cytokines as neuromodulators in cardiovascular control. Clin. Exp. Pharmacol. Physiol. 37, e52-e57 (2010).

28. Campese, V. M. et al. Reactive oxygen species stimulate central and peripheral sympathetic nervous system activity. Am. J. Physiol. Heart Circ. Physiol. 287, H695-H703 (2004).

29. Guggilam, A. et al. TNF-alpha blockade decreases oxidative stress in the paraventricular nucleus and attenuates sympathoexcitation in heart failure rats. Am. J. Physiol. Heart Circ. Physiol. 293, H599-H609 (2007).

30. Guggilam, A. et al. Cytokine blockade attenuates sympathoexcitation in heart failure: cross-talk between nNOS, AT-1R and cytokines in the hypothalamic paraventricular nucleus. Eur. J. Heart Fail. 10, 625-634 (2008).

31. Infanger, D. W. et al. Silencing nox4 in the paraventricular nucleus improves myocardial infarction-induced cardiac dysfunction by attenuating sympathoexcitation and periinfarct apoptosis. Circ. Res. 106, 1763-1774 (2010).

32. Kang, Y. M., Zhang, Z. H., Xue, B., Weiss, R. M. \& Felder, R. B. Inhibition of brain proinflammatory cytokine synthesis reduces hypothalamic excitation in rats with ischemia-induced heart failure. Am. J. Physiol. Heart Circ. Physiol. 295, H227-H236 (2008).

33. Ye, S., Zhong, H., Yanamadala, S. \& Campese, V. M. Oxidative stress mediates the stimulation of sympathetic nerve activity in the phenol renal injury model of hypertension. Hypertension. 48, 309-315 (2006).

34. Yu, Y. et al. Does aldosterone upregulate the brain renin-angiotensin system in rats with heart failure? Hypertension. 51, 727-733 (2008). 
35. Kannel, W. B. Hazards, risks, and threats of heart disease from the early stages to symptomatic coronary heart disease and cardiac failure. Cardiovasc. Drugs Ther. 11, 199-212 (1997).

36. Poulsen, S. H., Jensen, S. E., Gotzsche, O. \& Egstrup, K. Evaluation and prognostic significance of left ventricular diastolic function assessed by Doppler echocardiography in the early phase of a first acute myocardial infarction. Eur. Heart J. 18, 1882-1889 (1997).

37. Liao, Y. et al. Activation of adenosine A1 receptor attenuates cardiac hypertrophy and prevents heart failure in murine left ventricular pressure-overload model. Circ. Res. 93, 759-766 (2003).

38. Sugano, Y. et al. Granulocyte colony-stimulating factor attenuates early ventricular expansion after experimental myocardial infarction. Cardiovasc. Res. 65, 446-456 (2005).

39. Cho, E. et al. Role of inflammation in the pathogenesis of cardiorenal syndrome in a rat myocardial infarction model. Nephrol. Dial. Transplant. 28, 2766-2778 (2013).

40. Ye, J. et al. A new technique of coronary artery ligation: experimental myocardial infarction in rats in vivo with reduced mortality. Mol. Cell Biochem. 176, 227-233 (1997).

41. Lekawanvijit, S. \& Krum, H. Cardiorenal syndrome: acute kidney injury secondary to cardiovascular disease and role of proteinbound uraemic toxins. J. Physiol. 592, 3969-3983 (2014).

42. Salman, I. M. et al. Renal ischemic injury affects renal hemodynamics and excretory functions in Sprague Dawley rats: involvement of renal sympathetic tone. Ren. Fail. 32, 96-102 (2010).

43. Bauersachs, J. et al. Improvement of renal dysfunction in rats with chronic heart failure after myocardial infarction by treatment with the endothelin A receptor antagonist, LU 135252. J. Hypertens. 18, 1507-1514 (2000).

44. Darling, I. M. \& Morris, M. E. Evaluation of True Creatinine Clearance in Rats Reveals Extensive Renal Secretion. Pharm. Res. 8 1318-1322 (1991).

45. Brantsma, A. H., Bakker, S. J., de, Z. D., de Jong, P. E. \& Gansevoort, R. T. Extended prognostic value of urinary albumin excretion for cardiovascular events. J. Am. Soc. Nephrol. 19, 1785-1791 (2008).

46. Hallan, S. et al. Association of kidney function and albuminuria with cardiovascular mortality in older vs younger individuals: The HUNT II Study. Arch. Intern. Med. 167, 2490-2496 (2007).

47. Jackson, C. E. et al. Albuminuria in chronic heart failure: prevalence and prognostic importance. Lancet. 374, 543-550 (2009).

48. Hallan, S. I. et al. International comparison of the relationship of chronic kidney disease prevalence and ESRD risk. J. Am. Soc. Nephrol. 17, 2275-2284 (2006).

49. Dong, Z. et al. Myocardial infarction accelerates glomerular injury and microalbuminuria in diabetic rats via local hemodynamics and immunity. Int. J. Cardiol. 179, 397-408 (2015).

50. Arima, S. Role of angiotensin II and endogenous vasodilators in the control of glomerular hemodynamics. Clin. Exp. Nephrol. 7, $172-178(2003)$

51. Masson, S. et al. Elevated plasma renin activity predicts adverse outcome in chronic heart failure, independently of pharmacologic therapy: data from the Valsartan Heart Failure Trial (Val-HeFT). J. Card. Fail. 16, 964-970 (2010).

52. Kriz, W. \& Lehir, M. Pathways to nephron loss starting from glomerular diseases-insights from animal models. Kidney Int. 67, 404-419 (2005).

53. Silverberg, D., Wexler, D., Blum, M., Wollman, Y. \& Iaina, A. The cardio-renal anaemia syndrome: does it exist? Nephrol. Dial. Transplant. 18, viii7-12 (2003).

54. Silverberg, D. S., Wexler, D., Iaina, A. \& Schwartz, D. The interaction between heart failure and other heart diseases, renal failure, and anemia. Semin. Nephrol. 26, 296-306 (2006).

55. Bujak, M. et al. Interleukin-1 receptor type I signaling critically regulates infarct healing and cardiac remodeling. Am. J. Pathol. 173, $57-67$ (2008).

56. Gurantz, D. et al. IL-1beta and TNF-alpha upregulate angiotensin II type 1 (AT1) receptors on cardiac fibroblasts and are associated with increased AT1 density in the post-MI heart. J. Mol. Cell Cardiol. 38, 505-515 (2005).

57. Edner, M., Benson, L., Dahlstrom, U. \& Lund, L. H. Association between renin-angiotensin system antagonist use and mortality in heart failure with severe renal insufficiency: a prospective propensity score-matched cohort study. Eur. Heart J. 36, 2318-2326 (2015).

58. House, A. A., Haapio, M., Lassus, J., Bellomo, R. \& Ronco, C. Therapeutic strategies for heart failure in cardiorenal syndromes. Am. J. Kidney Dis. 56, 759-773 (2010).

59. Izuhara, Y. et al. Renoprotective properties of angiotensin receptor blockers beyond blood pressure lowering. J. Am. Soc. Nephrol. 16, 3631-3641 (2005).

60. Li, Y. L. et al. Angiotensin II enhances carotid body chemoreflex control of sympathetic outflow in chronic heart failure rabbits. Cardiovasc. Res. 71, 129-138 (2006).

61. Salman, I. M. et al. Role of the renal sympathetic nervous system in mediating renal ischaemic injury-induced reductions in renal haemodynamic and excretory functions. Pathology. 42, 259-266 (2010).

62. Stegbauer, J. et al. Angiotensin II modulates renal sympathetic neurotransmission through nitric oxide in AT2 receptor knockout mice. J. Hypertens. 23, 1691-1698 (2005).

63. Wilcox, C. S. Oxidative stress and nitric oxide deficiency in the kidney: a critical link to hypertension? Am. J. Physiol. Regul. Integr. Comp. Physiol. 289, R913-R935 (2005).

64. Wada, A. et al. Effects of a specific endothelin-converting enzyme inhibitor on cardiac, renal, and neurohumoral functions in congestive heart failure: comparison of effects with those of endothelin A receptor antagonism. Circ. 99, 570-577 (1999).

65. Whaley-Connell, A. et al. Comparative effect of direct renin inhibition and AT1R blockade on glomerular filtration barrier injury in the transgenic Ren2 rat. Am. J. Physiol. Ren. Physiol. 298, F655-F661 (2010).

66. Ye, S., Zhong, H., Yanamadala, V. \& Campese, V. M. Renal injury caused by intrarenal injection of phenol increases afferent and efferent renal sympathetic nerve activity. Am. J. Hypertens. 15, 717-724 (2002).

67. Zhang, M. et al. Endogenous hydrogen peroxide in the hypothalamic paraventricular nucleus regulates neurohormonal excitation in high salt-induced hypertension. Toxicol. Lett. 235, 206-215 (2015).

68. Huber, M. J. et al. Activation of the (pro)renin receptor in the paraventricular nucleus increases sympathetic outflow in anesthetized rats. Am. J. Physiol. Heart Circ. Physiol. 309, H880-H887 (2015).

69. Kang, Y. M. et al. Cross-talk between cytokines and renin-angiotensin in hypothalamic paraventricular nucleus in heart failure: role of nuclear factor-kappaB. Cardiovasc. Res. 79, 671-678 (2008).

70. Zhu, G. Q. et al. AT1 receptor mRNA antisense normalizes enhanced cardiac sympathetic afferent reflex in rats with chronic heart failure. Am. J. Physiol. - Heart Circulatory Physiology. 287, H1828-H1835 (2004).

71. Francis, J., Wei, S. G., Weiss, R. M. \& Felder, R. B. Brain angiotensin-converting enzyme activity and autonomic regulation in heart failure. Am. J. Physiol. Heart Circ. Physiol. 287, H2138-H2146 (2004).

72. Nakamura, K., Al-Ruzzeh, S., Ilsley, C., Yacoub, M. H. \& Amrani, M. Acute effect of cerivastatin on cardiac regional ischemia in a rat model mimicking off-pump coronary surgery. J. Card. Surg. 20, 507-511 (2005).

73. Liu, Y. H. et al. Chronic heart failure induced by coronary artery ligation in Lewis inbred rats. Am. J. Physiol. 272, H722-H727 (1997).

74. Suzuki, K. et al. Overexpression of interleukin-1 receptor antagonist provides cardioprotection against ischemia-reperfusion injury associated with reduction in apoptosis. Circ. 104, I308-I3I3 (2001) 
75. Adlam, V. J. et al. Targeting an antioxidant to mitochondria decreases cardiac ischemia-reperfusion injury. FASEB J. 19, 1088-1095 (2005).

76. Sammut, I. A. et al. Heat stress contributes to the enhancement of cardiac mitochondrial complex activity. Am. J. Pathol. 158, 1821-1831 (2001).

77. Lowry, O. H., Rosebrough, N. J., Farr, A. L. \& Randall, R. J. Protein Measurement with the Folin Phenol Reagent. J. Biol. Chem. 193, 265-275 (1951).

\section{Acknowledgements}

The authors would like to thank: A. Professor Dominic Parry Cardiothoracic Surgery, Dunedin Hospital for his assistance in training Scott Smart in the coronary artery ligation surgery, Mrs Mandy Fisher, Histology Unit for her assistance in the preparation of tissues for histological analysis and Clare Fitzpatrick, Department of Microbiology for her assistance with the Luminex $100^{\mathrm{TM}}$ analyser. This work was supported by equipment grants from the National Heart Foundation of New Zealand and New Zealand Lottery Health to JCH, IAS and scholarships from the Health Research Council NZ and Otago Medical Research Fund to SDGS for which we are most thankful.

\section{Author contributions}

Study conception \& design I.A.S., J.C.H. \& S.D.G.S., student training \& supervision I.A.S. \& J.C.H., coronary artery ligation surgery and cardiac perfusion S.D.G.S., tissue collection, preparation \& biochemical analysis S.D.G.S., J.C.H., E.H.B., J.R.K., Y.Y. \& M.I.R., histology S.D.G.S., E.H.B., J.R.K. \& M.I.R., data analysis I.A.S., S.D.G.S., J.C.H., E.H.B., J.R.K. \& Y.Y., preparation of figures for publication S.D.G.S., J.C.H., E.H.B. \& J.R.K., drafting of sections of manuscript S.D.G.S., J.C.H., E.H.B., Y.Y. \& J.R.K., drafting main manuscript J.C.H., critical evaluation and editing manuscript J.C.H., M.I.R. \& I.A.S., final approval of the manuscript I.A.S., S.D.G.S., J.C.H., M.I.R., E.H.B., J.R.K. \& Y.Y. I.A.S. is the guarantor of this work and, as such, has full access to all of the data in the study and takes responsibility for the integrity of the data and the accuracy of the data analysis.

\section{Competing interests}

The authors declare no competing interests.

\section{Additional information}

Supplementary information is available for this paper at https://doi.org/10.1038/s41598-020-58071-x.

Correspondence and requests for materials should be addressed to J.C.H.

Reprints and permissions information is available at www.nature.com/reprints.

Publisher's note Springer Nature remains neutral with regard to jurisdictional claims in published maps and institutional affiliations.

(c) (i) Open Access This article is licensed under a Creative Commons Attribution 4.0 International License, which permits use, sharing, adaptation, distribution and reproduction in any medium or format, as long as you give appropriate credit to the original author(s) and the source, provide a link to the Creative Commons license, and indicate if changes were made. The images or other third party material in this article are included in the article's Creative Commons license, unless indicated otherwise in a credit line to the material. If material is not included in the article's Creative Commons license and your intended use is not permitted by statutory regulation or exceeds the permitted use, you will need to obtain permission directly from the copyright holder. To view a copy of this license, visit http://creativecommons.org/licenses/by/4.0/.

(C) The Author(s) 2020 